\title{
An Empirical Study on Interactive Flipped Classroom Model Based on Digital Micro-Video Course by Big Data Analysis and Models
}

\author{
Na Tian ${ }^{1}{ }^{1}$ and Sang-Bing Tsai $\mathbb{D i}^{2}$ \\ ${ }^{1}$ Gingko College of Hospitality Management, Chengdu 610000, China \\ ${ }^{2}$ Regional Green Economy Development Research Center, School of Business, Wuyi University, Nanping, China
}

Correspondence should be addressed to Na Tian; tinna8296134@163.com

Received 8 October 2021; Revised 26 October 2021; Accepted 10 November 2021; Published 25 November 2021

Academic Editor: Mingwei Lin

Copyright (C) $2021 \mathrm{Na}$ Tian and Sang-Bing Tsai. This is an open access article distributed under the Creative Commons Attribution License, which permits unrestricted use, distribution, and reproduction in any medium, provided the original work is properly cited.

\begin{abstract}
This paper provides an in-depth analysis and study of the interactive flipped classroom model for a digital micro-video for a big data English course. To improve the learning efficiency of English courses and reduce the learning pressure of students, the thesis also uses certain techniques to apply audiovisual language to the production of specific micro-class videos, broadcast the successfully recorded micro-class courses to students, and then use the questionnaire to randomly distribute the designed audiovisual language use questionnaire. Micro-classes earnestly perform data statistics for students and finally conduct data analysis to summarize and verify the effects of micro-class audiovisual language use. The improved algorithm can effectively reduce the fluctuation of the consumption of various resources in the cluster and make the services in the cluster more stable. The new distributed interprocess communication based on protocol and serialization technology is more efficient than traditional communication based on protocol standards, reduces bandwidth consumption in the cluster, and improves the throughput of each node in the cluster. The content design and scripting of micro-video teaching resources are based on this. Then, the production process of micro-video teaching resources is explained, according to the selection of tools, the preparation, recording, editing, and generation of materials.
\end{abstract}

\section{Introduction}

With the large-scale promotion and use of information technology and network technology, video data have taken up $70 \%$ of the Internet traffic. In the field of life, short video and real-time live streaming are becoming increasingly popular; in the field of surveillance, network surveillance cameras are spread all over the city; in the field of office, cloud video conferencing is also gradually approaching people's work. In the future, there will be increased video data, and these video data have high timeliness. Unlike text information, video data have multidimensional, large volume, high hidden characteristics; compared with text data, video data are more complex and require higher computational requirements [1]. It is inefficient to analyze the cameras in urban corners by hand alone, so how to mine the information in these unstructured data through computer technology in time to further improve the work productivity has also become a major challenge for enterprises and related research institutions [2]. However, these frameworks have been developed over the years, and to make the frameworks have better generality, the community developers have not made optimization for specific scenarios (e.g., video processing), and users need to optimize the configuration according to their business scenarios and modify the source code if necessary [3]. At the same time, according to the theory and practice of micro-video teaching, there is a natural combination of micro-video 
teaching and scaffolded teaching, and the combination of the two will significantly improve the teaching efficiency of English listening classroom. In terms of specific practices, first, complex learning tasks can be decomposed through scaffolded teaching to gradually guide learners to deeper understanding. Then micro-videos are used in each level of the decomposed tasks, allowing students to improve their listening skills by watching them and creating a contextualized listening learning atmosphere.

On the one hand, because the micro-course is composed of streaming video, the aesthetic standard of video audiovisual language is applicable; on the other hand, the use of film and television works with a large number of rich audiovisual language to shoot the storyline of the microcourse; the audiovisual language in line with the human visual and auditory sense helps students learn micro-course knowledge. It is an important research topic to apply the audiovisual language filming method in micro-course production to improve students' learning effect and highlight the focus of teaching content knowledge [4]. With the rise of the concept of flipped classroom teaching and the rapid development of large-scale open online courses, micro-courses are also becoming another breakthrough in the exploration of educational reform. The advent of the "micro" era has affected people in every aspect. With the rapid development of network communication technology, micro-courses are also developing rapidly, and with their short characteristics, they effectively solve the micro-needs of learners. Through theoretical research and comparative analysis of the audiovisual language elements in the excellent micro-course videos and comparison with the rich audiovisual language in film and television works, the study of the unique audiovisual language in micro-courses and the search for the use of audiovisual language in micro-course production can provide some reference to reduce the fatigue of learners in long micro-courses.

The importance of learning English grammar is selfevident. However, from my own teaching experience and the current situation of local students learning English, the teaching and learning of English grammar are not particularly satisfactory [5]. First, due to the heavy workload and the reform of English examination papers, some teachers and students have the concept of "light on grammar, grammatical plates and light on special sentences," which is manifested by the fact that everyone pays more attention to big grammar, such as the three main clauses, verbs, and nonverbal verbs, and marginalizes special sentences. The first relevance of this study is to emphasize the importance of special sentences in grammar learning; even though microvideos and flipped classrooms have emerged in recent years, their effectiveness is still unknown, and people are afraid to implement them. Therefore, the second relevance of this study is to provide a reference for teachers who want to make a change in teaching special sentences as grammar, especially for those who have accumulated many high-quality grammar micro-videos in provincial micro-video competitions, and want to implement them specifically in their teaching. Under the concept of the flipped classroom, students learn by watching the micro-video first, then answer questions, and consolidate their knowledge through discussion or asking the teacher for advice; students' initiative can be strengthened, and students' deep understanding of grammar knowledge can be achieved; and students' main position can be highlighted, and students can use the fragmented time to learn everywhere, which can free up more time in class to strengthen.

\section{Current Status of Research}

To facilitate this, the videos were posted online for their independent learning, and as the number of hits increased, he began to think about the advantages of this teaching method. Inspired by the videos, many teachers have tried to change the way they taught in the classroom by having students watch the videos at home before class to grasp the main points of the lesson and to assess their proficiency through appropriate exercises; then in class, they focus on problem-solving and collaborative communication between the teacher and the students. Prensky et al. mentioned that with the development of science, technology, and the Internet, this generation of youth is different from their parents' generation [6]. In the face of this explosion of knowledge, although some people complain about their concentration and attention span, others argue that it is not because they are not concentrating but because they have more options, their attention span has changed, and their needs are different [7]. There are so many things that appeal to them in the face of a colorful world. Faced with this world of light and darkness, it is no longer possible for them to be as simple as the past generation, and their independent thinking and individuality gradually emerge [8]. Education, then, must be branded with the digital age to capture and engage students, and Salman Khan and others mention that there is both serendipity and inevitability in being an early researcher in the flipped classroom [9]. The serendipity lies in the fact that he did not realize that he would have such a big impact until then. He simply hoped to use his recorded instructional videos to tutor his relatives on their homework, but he did not expect to receive good results. The necessity lies in the fact that as an education practitioner, he has always believed that what exists may not be justified. The drawbacks of traditional education, which have become apparent, cannot ignore just because people have become accustomed to its existence [10].

The analysis and collation of the scholars' understanding of flipped classrooms show that everyone's understanding of flipped classrooms is similar, based on the development of science and technology, the catalyst of the change of knowledge learning mode, plus the escort of independent learning [11]. Combined with the analysis and collation, the author then synthesizes his own practical experience and believes that the existence of flipped classrooms has its inevitability. For students at a better level like the one the author is dealing with, one will find that the current generation is different from the previous one in many ways [12]. The previous generation may not know what big data, 5G, and the Internet are; they already live in a smart city and feel them everywhere; and they often scoff at some shallow 
understanding and memorization [13]. Sometimes, within the class, while the teacher drones on about some grammar rules, many students are already off to do their own thing [14]. For them, flipping the classroom is not a bad way to learn. Because it realigns the time inside and outside the classroom, the teacher no longer has the power to make decisions about learning, and instead, it is transferred to the students. With this model of teaching and learning, the valuable time within the classroom, students can focus more on active project-based learning, working together to solve problems and thus gain a deeper understanding [15].

It can effectively reduce the pressure on the server, but it will increase the requirements for the hardware configuration of the user's device, and if the configuration is too low, the user experience will be greatly reduced. At the same time, this approach can only integrate some lightweight algorithms; otherwise, it will increase the requirements for the user's terminal device configuration and even cause hardware loss. After years of development, the stream computing framework has become increasingly complex, although it has become richer and richer in functions, and the technology is maintained by large enterprises, and SMEs or research institutions do not have enough resources to maintain it, and it is difficult to troubleshoot problems [16]. Moreover, the development of current stream computing frameworks is mainly borrowed from the Internet, and there is a lack of product-tested stream computing frameworks for real-time video stream data processing scenarios. All three solutions for video stream processing have their applicable scenarios, and different R\&D vendors will adopt different strategies to improve user experience. However, the greatest scope for development is the clustering of video streams, which is a key research direction for companies with video businesses today. The method of clustering processing has no upper limit on the throughput rate and processing delay in theory, and the size of the throughput rate can be controlled by dynamically adjusting the number of service nodes in the cluster, and the cluster can be flexibly adapted to various scenarios through intelligent dynamic configuration.

\section{A Study on Interactive Flipped Classroom Model for Big Data English Course with Digital Micro Video}

\subsection{Big Data Platform Design for Digital Micro-Video.} First, we need to design and implement the basic functions of this video big data intelligent analysis platform, mainly including five modules, which are: real-time statistics, real-time monitoring, query retrieval, resource management, and system settings. Some of the function modules contain some subfunctions; query retrieval subfunctions include: historical alarms, capture retrieval, 1:1 comparison, and personnel database comparison; resource management subfunctions include: personnel database management, control management, and bayonet management; and system settings subfunctions include: department management and user management. In addition to the basic functions of the system, based on the capture records in the system, it is also necessary to implement some functions of target person analysis [17]. When the number of cameras deployed in the area reaches a certain level, personnel behavior analysis can be analyzed. The system architecture design is the beginning of developing a software system and is also crucial to the implementation and performance of the system. Properly designed architecture not only allows for smooth and efficient development of subsequent functions but also allows for more stable operation during the operational phase of the system, saves physical resources such as servers, and has higher availability and fault tolerance. In addition, when facing changes in requirements, a good architecture makes it easier for the system to cope with these changes so that it can bring more adaptation with fewer changes. It can be said that architecture design is the top priority in software system development. When implementing this video big data intelligent analysis platform, we need to fully consider the stability and scalability of the system to ensure that the system can run stably for a long time, and it is also best to upgrade the system without interrupting the services provided to the public [18]. When adding cameras to the system, both the processing of video and face data or the relevant capture records and pictures of storage will increase the burden on the system; at this time, the system scalability is also very important. To ensure that when adding real-time video to the system for analysis, the system operation is not interrupted, and the service is not interrupted, so the system needs to have the ability to dynamically expand the capacity.

Micro-video teaching resources should have a scientific process as the mainline of production, according to which the production process is concretized. The production of micro-video teaching resources includes the selection of tools, preparation of materials, and a series of recording, editing, and finally generating the video format required for teaching. Micro-video teaching resources should have a scientific process as the mainline of production, according to which the specific production process is launched. The production of micro-video teaching resources includes the selection of tools, the preparation of materials, and a series of recordings, editing, and the final generation of the video format required for teaching. This is the work of collecting and preparing materials such as pictures, videos, audios, and PPT courseware needed in the stage of making micro-video teaching resources, which is related to the specific recording practice in the next stage, so it is necessary to be well prepared. When collecting materials, we should take the needs of learners as the primary principle, consider the cognitive development of secondary school learners, and try to focus on interesting and popular materials, to be easy to understand and unique [19]. In the cluster, if a new node appears, to prevent the new node from scheduling too many nodes, we will monitor the scheduling success event of the scheduler, obtain the scheduling result, and mark the number of scheduled pods for each node in the past period, such as the number of pods scheduled within $1 \mathrm{~min}, 5 \mathrm{~min}$, and $30 \mathrm{~min}$, the hotspot value of the node is measured and 
then compensated to the node's preference score, so this will become increasingly stable. This is the stage of concretizing the practice of micro-video teaching resources. The author uses the pre-selected tools for recording work, and editing can be done within the recording software or using other post-editing tools to insert the required images, audio, animation, and so on. After finishing the editing work, the required format is generated, and if there are special format requirements, format conversion can be done using Format Factory software. After the initial completion, the microvideo teaching resources are shown to some learners; the viewing experience is collected; the resources are adjusted and improved according to other teachers' suggestions; and the content and form of the micro-video teaching resources has to be continuously optimized until the final version is generated, to obtain the desired teaching effect in the practice stage of the resources, as shown in Figure 1.

Since a cluster environment is more complex compared to a standalone environment and the cluster contains various resource conditions that affect each other, a distributed processing system needs to be able to not only complete the tasks submitted by users but also dynamically balance the cluster load and rationally allocate cluster resources so that the efficiency of task processing in the cluster can be improved. We can optimize the cluster load by intelligently scheduling tasks through load balancing algorithms. Allocation and scheduling of tasks in a distributed system require the use of load balancing algorithms. The load balancing algorithm studied in this paper will combine the weighted voting method based on real-time resource monitor and system load prediction by deep learning-based time series prediction algorithm so that the tasks can be allocated reasonably. Since a cluster environment is more complex compared to a standalone environment and the cluster contains various resource conditions that affect each other, a distributed processing system needs to be able to not only complete the tasks submitted by users but also dynamically balance the cluster load and rationally allocate cluster resources so that the efficiency of task processing in the cluster can be improved. We can optimize the cluster load by intelligently scheduling tasks through load balancing algorithms (see Figure 1).

Allocation and scheduling of tasks in a distributed system require the use of load balancing algorithms. The load balancing algorithm studied in this paper will combine the weighted voting method based on real-time resource monitor and system load prediction by deep learning-based time series prediction algorithm so that the tasks can be allocated reasonably.

The weighted voting method is to assign a weight value to each condition in advance. Let there be $n$ conditions related to the resource consumption of the service node and each resource is given a weight value. Then the final service computing node's resource consumption can be calculated by the following equation:

$$
P=\sum_{i=0}^{n} P_{i} \times W_{i}^{2} .
$$

The advantage of performing load balancing in this way is that the hardware configuration of each node in the cluster can vary, as the final task is assigned based on the percentage of available resources to the total resources. The dynamic load balancing algorithm designed in this paper does not rely completely on load prediction algorithms, as there are no accurate prediction algorithms available. The load balancing algorithm starts by obtaining the current resource utilization status of each service node in the cluster, then predicts the candidate nodes, selects the node that is least likely to generate a resource utilization spike in the future period, and finally assigns the task to that node.

$$
\begin{aligned}
P_{b} & =\frac{\left(\sum_{i=1}^{n} T+\sum_{i=1}^{n} R\right)}{(n \times I \times T b)}, \\
R & =P_{b} \times W_{b}-P_{m} \times W_{m}-P_{c} \times W_{c} .
\end{aligned}
$$

From the functions of each part of the system, we can see that the system is closely focused on face data, and many functions are closely related to capture records or dynamic face data. For example, the alarm is judged according to the dynamic face capture data, and the functions such as trajectory analysis, analysis of the same pedestrian, analysis of the landing point, and frequent passing are also based on the analysis and calculation of the capture records. Face capture records are very important to the whole system. In addition, as the number of cameras connected to the system increases, the number of face captures will also increase, and the dynamic face data that needs to be processed by the system in real-time will also increase. If the dynamic face data is not processed properly, the light will make the capture retrieval, alarm, and other functions delayed and heavy perhaps with the increasing number of data to be processed, until the whole system dragged down. Therefore, dynamic face data processing can be said to be the top priority in the whole system implementation process, as shown in Figure 2.

After collecting the data through TensorFlow's datacleaning interface, it is possible to separate the data set into test data (20\%) and training data (80\%); since the data are temporally attributed in this scenario, there is no mashing of the data, and parameters need to be introduced in this interface to get the same structure for all the same inputs.

$$
\begin{aligned}
p_{t}^{\prime} & =\frac{p_{t}+\max (p)}{\max (p)+\min (p)}, \\
W_{t} & =\left(p_{t-w}^{1}, p_{t-w}^{2}, p_{t-w}^{3}, \ldots, p_{t-w}^{w}\right), \\
p_{t}^{w} & =f\left(p_{t-w}^{1}-p_{t-w}^{2}+p_{t-w}^{3}-p_{t-w}^{w}\right) .
\end{aligned}
$$

Students' interest in learning is the one with the highest mean among the four dimensions, from which students are more inclined to agree that micro-video scaffolded instruction can increase students' interest in learning (see Figure 2).

In addition, the standard deviation of the effect of microvideo scaffolded instruction on students' interest in learning is 0.71505 lower than the standard deviation of the other 


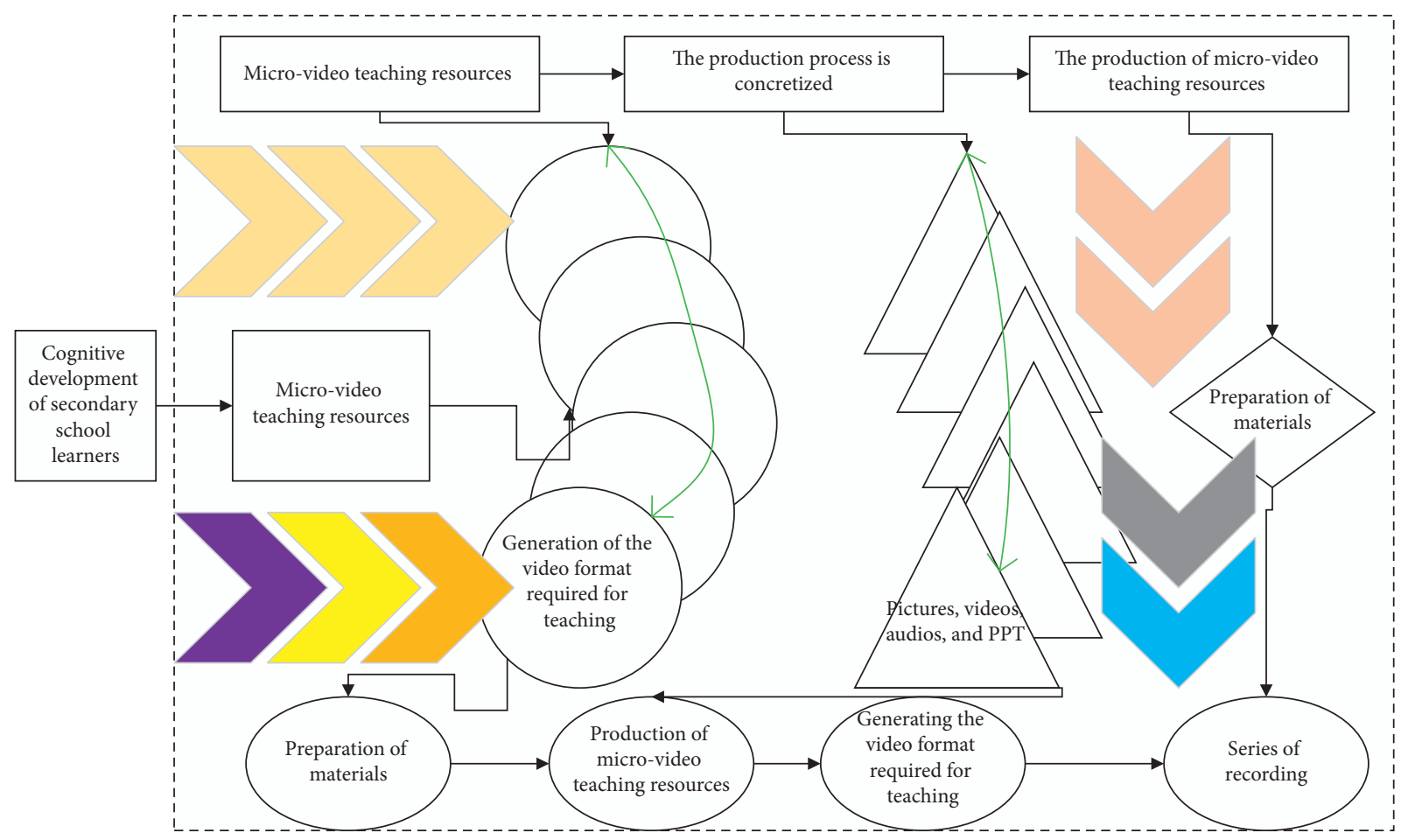

FIGURE 1: Stream computing architecture.

three dimensions, which leads to the conclusion that students are more inclined to agree that micro-video scaffolded instruction has the effect of increasing interest in learning.

$$
3 \leq W \leq W_{\min } .
$$

It can be seen that the model-based prediction algorithm has some accuracy in predicting the overall trend of the load situation, but it also has some error, so the final load balancing algorithm does not fully rely on this prediction algorithm, and then when assigning tasks, it will first discard the part of nodes with heavier load calculated by the prediction algorithm, then obtain the load situation of the remaining cluster nodes through the dynamic load balancing algorithm, and finally assign the tasks to the service nodes with the lowest load.

3.2. Design of an Interactive Flipped Classroom Model for English Courses. The mechanism of the more mature Kafka has been thoroughly studied, and our research goal is not the mechanism of Kafka. The connection of the three modules in the flipped classroom model is done through information technology; without the support of information technology, the flipped classroom model cannot be implemented. IT is a powerful lever for instructional designers to create a learning environment for students [20]. IT makes it possible for teachers to track and supervise students' learning online, to guide and help them online, to test and evaluate their learning results online, and to ensure personalized independent learning and collaborative group learning for students. The self-study and questioning stages are entirely at the students' own pace. First, the subject class representative will lead the interpretation of the learning objectives and arrange the learning tasks, and students will follow the learning objectives for self-study of the material. Students can read the textbook first to master the knowledge content and then complete the study plan independently, or they can read the textbook according to the study plan questions and then complete the study plan independently. The difficult problems encountered in the process of reading the textbook on their own can be studied with the help of micro-lessons recorded by the teacher in advance. The micro-lesson can be paused and rewatched repeatedly, and the problems in the lesson plan can be improved and revised after reading and understanding. After the micro-lesson, there are still questions that are not understood, or new questions arise, which need to be discussed and exchanged among group members. Generally, the students who finish the learning task first to communicate can be followed as models, and the communication experience is shared among the group members or the group. Of course, there may be questions that students do not understand or additional questions that arise, and students will need to write these down on a "question feedback slip." In addition, students will need to complete online tests prepared by the teacher in advance to test their learning. Teachers need to collect and summarize students' questions, data from the platform, and feedback from the learning plan. The teacher will design the breakthrough points and personalized tutorials after fully understanding the students' learning situation.

The micro-video-based flipped classroom teaching model should include three components: pre-class, in-class, and post-class. The components of the pre-lesson session include teacher's production of teaching micro-video, 


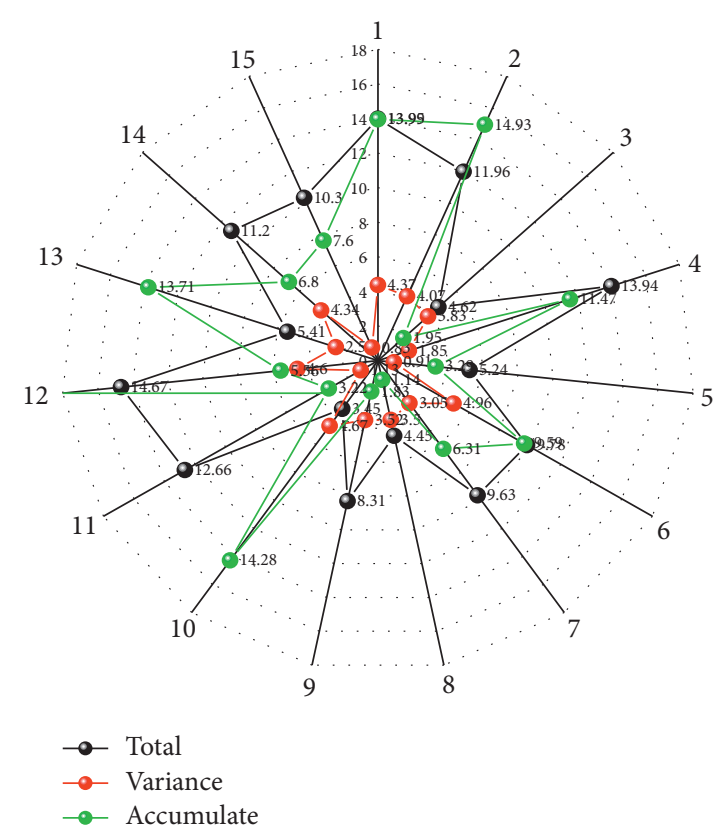

FIgURE 2: Explanation of the total variance of the factors.

teacher's design of pre-lesson independent learning task list, students' viewing of teaching micro-video, students' completion of the pre-lesson independent learning task list, students' marking of doubts, asking questions, and so on. The components of the midlesson session include warm-up of learning contents, students' independent work, group collaborative learning, display of learning results, teacher's guidance and counseling, teacher's evaluation, and feedback. The components of the post-lesson session include students' production work, students' sharing of work, teachers' evaluation of work, students' mutual evaluation of work, midterm, final exams, and so on. On this basis, the author combines the theory of ubiquitous learning, the theory of associationist learning and proficiency learning, as well as the theory of motivation, and constructs a flipped classroom teaching model based on micro-video, as shown in Figure 3.

The biggest difference between micro-video-based flipped classroom teaching mode and traditional classroom teaching is the part of knowledge transfer. In traditional classroom teaching, knowledge transfer is in the middle of the class, while micro-video-based flipped classroom teaching is done before the class, and the tasks of teachers and students change before the class. In traditional classroom teaching, the teacher's task is to remind students to preview what they are going to learn, with no specific instructions on how to preview and how to preview, and when students preview, they only preview the material, or even some students do not preview at all, causing the pre-class preview session to become a bubble. There are many tasks that teachers and students need to do in the pre-class session of micro-video-based flipped classroom teaching. First, teachers should make the micro-video a week before and distribute the video to students 2-3 days before; students get the micro-video and independent learning task list and then do independent learning before class; teachers should keep online guidance and supervision; students mark the problems they encounter when doing independent learning; and finally, teachers need to collect students' feedback and suggestions (see Figure 4).

Teaching evaluation refers to the process of measuring and value judgment on the process of teaching activities and their results following certain teaching objectives and using scientific and feasible standards and methods. Two points can be seen from the above definition: First, teaching evaluation is carried out following teaching objectives, and clear teaching objectives are the prerequisite for teaching evaluation. Therefore, the classification theory and method of teaching objectives and the development and changes of contemporary educational objectives concept dominate the process and method of teaching evaluation, developing their cognitive ability and the ability to criticize and create beauty mastering the systematic basic knowledge of culture and science, forming basic skills and techniques, developing their cognitive ability students' bodies to get normal development and healthy growth, gradually forming correct aesthetic views and the ability to feel the beauty and appreciate the beauty and create beauty, and gradually developing socialist moral behavior and ideological quality, the basis for the formation of a materialist worldview, and the comprehensive and harmonious development of their personalities [21]. Its factors are very rich, and teaching evaluation should be based on many factors that respond to the teaching objectives, as shown in Figure 4.

What teachers should do in this feedback session is to get questions and feedback to adjust their micro-video based on the effectiveness of the students' viewing of the instructional micro-video. Based on students' suggestions and comments on the instructional micro-video, the style, technique, format, and effectiveness of their instructional micro-video should be adjusted or changed. Students will record what 

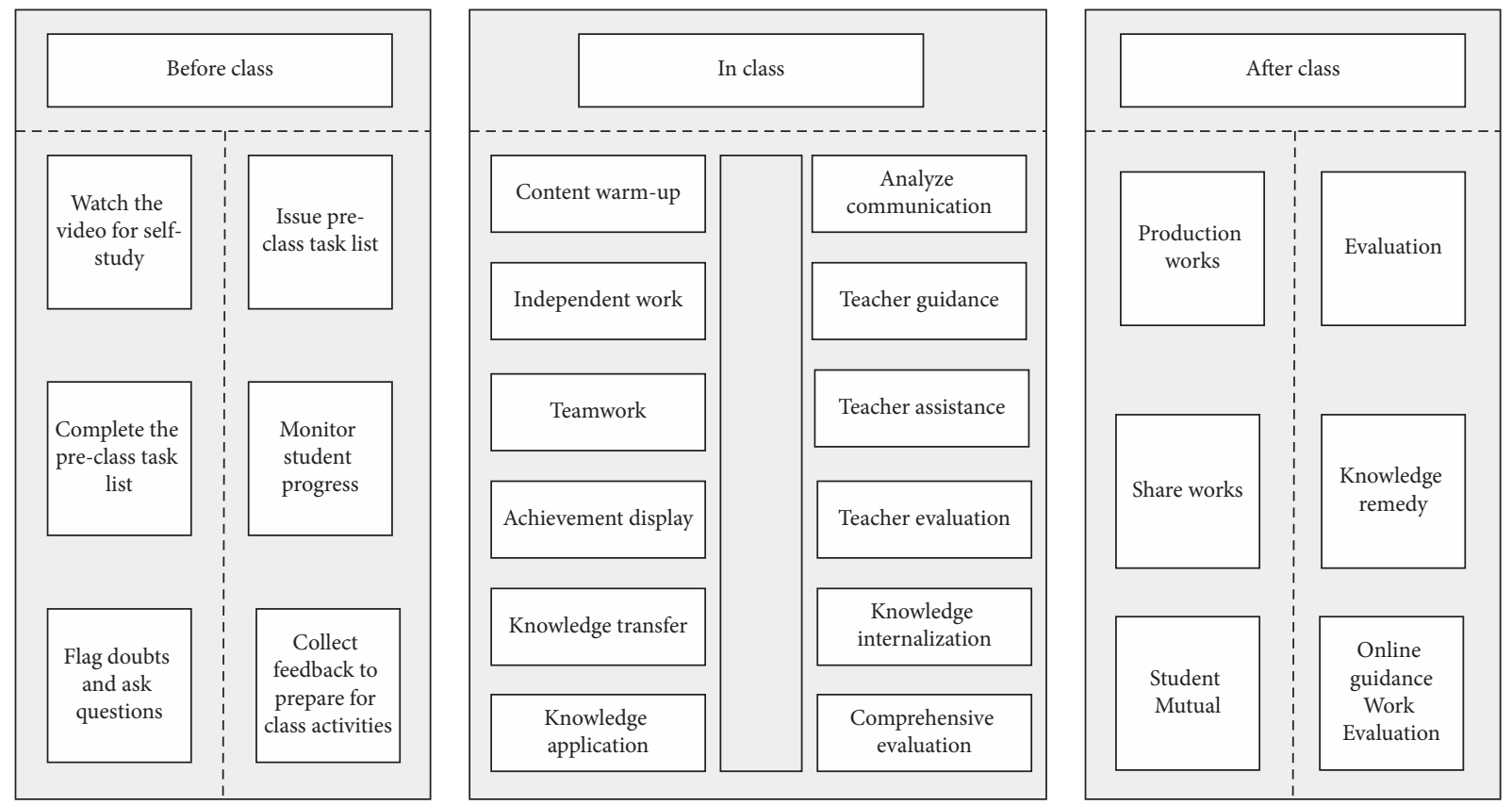

Figure 3: Micro-video-based flipped classroom teaching model.

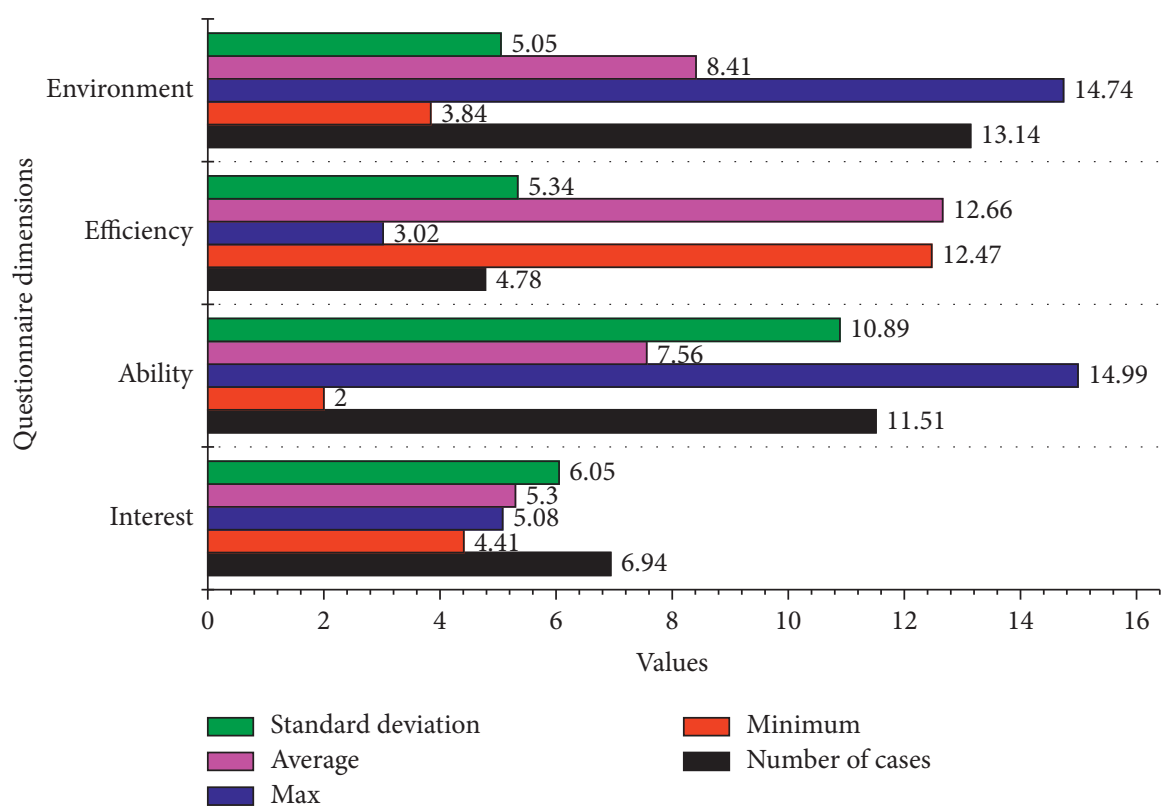

FIGURE 4: Impact of micro-video scaffolded instruction in the listening classroom.

they understood, what they did not understand, and what they want to study in-depth when they watch the instructional micro-video, and teachers will collect this feedback to prepare their in-class activities. In addition, teachers should create a comprehensive reward mechanism to increase students' enthusiasm and motivation by offering rewards for good performance, such as students who watch instructional micro-videos particularly attentively and who ask good questions or suggestions.

The students in the researcher's school have no problem in mastering basic grammar, such as lexis, tenses, and so on.
Among the syntax, they have a good grasp of the frequently used and important definite clauses, but they have some difficulty in learning other macro-sentence types, such as noun clauses, and $90 \%$ of them cannot understand special sentences, which shows that the author's previous analysis of the students' learning situation is right.

\section{Analysis of Results}

4.1. Big Data Platform Performance Results for Digital MicroVideo. As the static load balancing algorithm does not 
consider the difference in the performance configuration of the service nodes in the cluster, it assigns tasks without any difference, resulting in node 3 reaching a bottleneck due to its performance, and the load situation is close to $100 \%$ at first, at which time node 3 can no longer accept tasks, but the computing resources of nodes 1 and 2 are still very sufficient at this time. Next, we use the same simulation to test the dynamic load balancing algorithm based on real-time resource monitoring; the test results are shown in Figure 5; from Figure 5, we can see that the overall resource consumption of the three service nodes is on the rise; although the fluctuation of no. 3 is relatively large (mainly due to the lack of CPU performance), there is no CPU burst when using the static load balancing algorithm. The dynamic load balancing algorithm gives priority to assigning tasks to the remaining nodes when node 3 has the highest resources, and the use of a dynamic load balancing algorithm can generally ensure the stable operation of the cluster (see Figure 5).

After applying the prediction-based load balancing algorithm, compared to the dynamic load balancing algorithm, the resource consumption of each node in the cluster becomes relatively smooth in general without particularly large fluctuations, except that the resource consumption of assigned tasks is relatively obvious due to the relatively poor performance of node 3 , the other two nodes are relatively smooth, and when a load of a certain machine is relatively large, the algorithm will assign tasks to other nodes, so the node resource consumption rises for a while and then drops significantly.

Considering the efficiency of distributed interprocess communication in different scenarios, this paper adopts two middleware communication methods, message queue, and serialized network communication and uses different serialization algorithms and compression algorithms for messages with different attributes to further improve the throughput and transmission efficiency. The message queue has already had a more mature Kafka, so this paper does not do a deep study on this. The choice of the communication method between service nodes is crucial in distributed stream computing application scenarios. In this paper, based on the traditional remote procedure call method, we improve it for the latest HTTP 2.0 standard to improve the overall throughput rate and transmission efficiency of distributed applications.

The interface was tested before and after the optimization of the capture count to verify the impact of placing the count results in the Redis cache on the performance of the interface. The response time of the interface at different levels was recorded in the test database, and the system was warmed up by running the test 100 times before the test and then tested 100 times at each level, recording the time taken for each call and taking the average. The test data is shown below: this experiment is to simulate the communication process of two distributed processes in a cluster with two servers, by sending a large amount of image data simultaneously from one server to another server using the traditional network communication method and our designed serialized network communication method, respectively, and compare the difference in communication efficiency between the two; the experimental results are shown in Figure 6.
From the experimental results, we can see that the traditional protocol-based network communication method is less efficient than the protocol-based serialized network communication method in the scenario of transmitting a large amount of binary data, and the larger the data size, the more obvious the advantage of this serialized network communication method. And in the case of more cameras in the system access, the number of capture records generated in the system every day may reach millions. And then with the accumulation of time, the total number of captures in the system may reach tens of millions or even hundreds of millions. At this time, the database to do statistics on such a huge number of records will undoubtedly be slow. Once the interface response speed is full to a certain extent, the overall performance of the system may be affected.

\subsection{Results of the Interactive FlippedCmodel for English} Courses. For computer application courses, students' proficiency in hands-on operation is poor, and students hope that the time allocated for computer operation is greater than the time taught by teachers in class, so that students can have more opportunities for independent practice, which is consistent with the concept of "students are the main body of learning" in the above micro-video-based flipped classroom teaching model. From Figure 7, only $15.0 \%$ of the students like or like very much the current traditional teaching classroom; $85.0 \%$ of the students think that the current traditional teaching model is average or dislike or dislike very much; moreover, only $11.0 \%$ of the students say that the current traditional teaching mode can stimulate their learning interest; and as many as $89.0 \%$ of the students think that the traditional teaching mode cannot stimulate their learning interest well. This shows that students expect a new teaching model based on the traditional teaching mode, and this mode should make students more interested in learning and increase their motivation to learn. Although $88.0 \%$ of the students did not understand the concept and implementation process of the flipped classroom, $87.0 \%$ of the students were more interested in learning about the flipped classroom; $79.0 \%$ of the students were more or very interested in the "flipped classroom" of this course; $97.0 \%$ of the students expressed their willingness to experience the flipped classroom teaching mode; and $46.0 \%$ of the students were interested in the "flipped classroom" of this course. The students were able to have a correct understanding of the meaning of "flipped classroom."

After independent work and exploration, students have constructed their cognitive structure or knowledge system, but to complete the deep internalization of knowledge, students need to communicate and help each other. Therefore, communication and mutual assistance among group members are needed in a session, and students who have completed three topics can help other members of the group. This kind of peer-to-peer communication and mutual help helps students dissect and explain the knowledge points and helps group members who did not find the correct answers at the beginning sort out their questions to form the correct perception. At the 


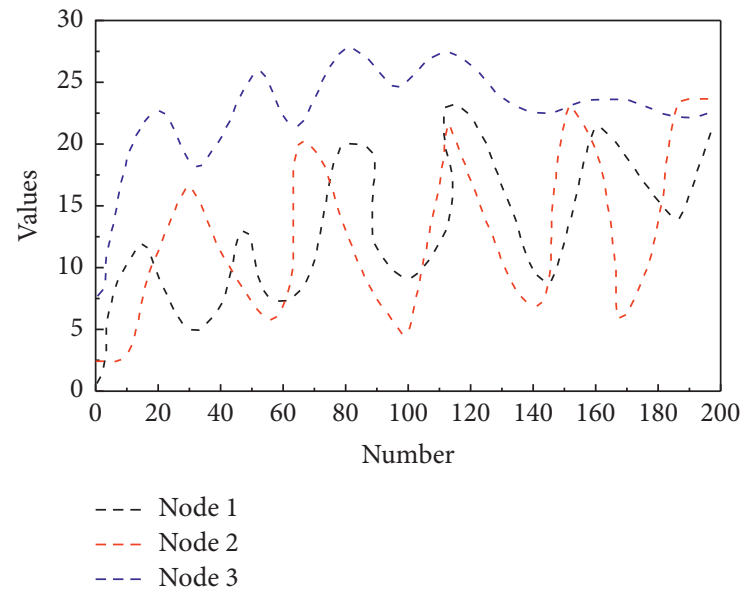

Figure 5: Dynamic load balancing.

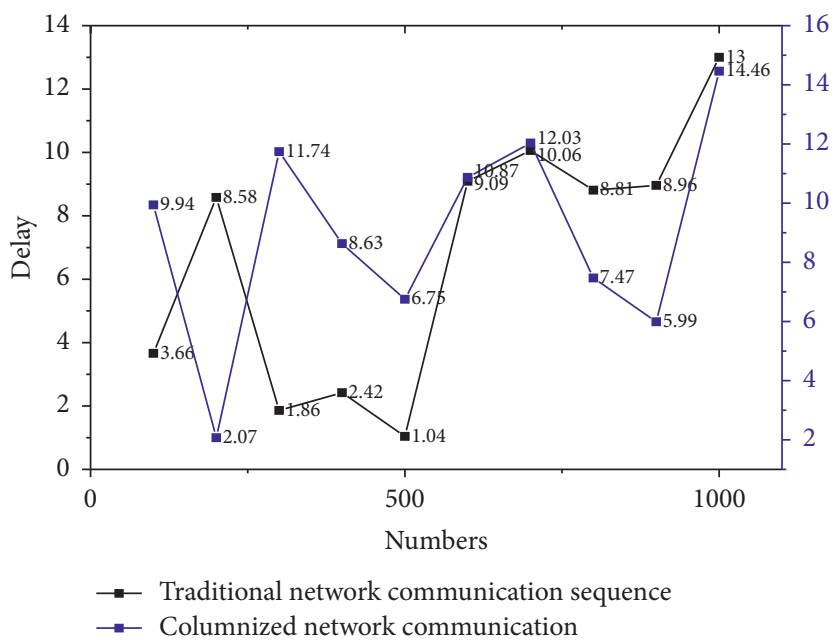

FIGURE 6: Experimental results of comparison of communication methods.

same time, the author will also join the students' group discussions to give timely guidance and help clarify students' misconceptions.

Students will develop their own or group learning outcomes after independent and collaborative group learning. In the above two sessions, what problems students solved and what problems remained unsolved, each group sends a representative out to present their learning outcomes and ask their questions. The presentation is done by the live demonstration method, where each group's representative goes to the teacher's machine and demonstrates the group's results to all the students, and the author evaluates the level of each group's demonstration and demonstrates results, and the groups evaluate each other (see Figure 7).

In Figure 7, what we show is the specific value under different headings. Due to a large number of types, this method is used for visual analysis. We can see that the values under different headings are not related in any way. As can be seen from Figure 8, after the micro-video-based flipped classroom teaching, the students in the experimental class preferred and liked the micro-video-based flipped classroom teaching model, which was more favored by the first-year students than the traditional teaching model. The results of the analysis showed that the overall mean value of the learning effectiveness section was 4.1543 , which was higher than the value of 4 for the "meet" option on the five-point scale, with a standard deviation of 0.1392 and variance of 0.0190 , indicating that the respondents generally believed that the use of micro-video teaching resources in the classroom had a positive impact on their ability to improve. The learners' hands-on, self-learning, and problem-solving skills were significantly improved, further explaining the reason for the relatively high overall performance of the learners. The question with the lowest score was "I think the use of micro-video teaching resources helps to enhance my observation and analysis skills," with a score of 3.9787, indicating that micro-video teaching resources have yet to be improved in enhancing learners' observation and analysis skills (see Figure 8).

Second, the sound effects can be used to cut the knowledge chapters, so that students can better appreciate the micro-course. 


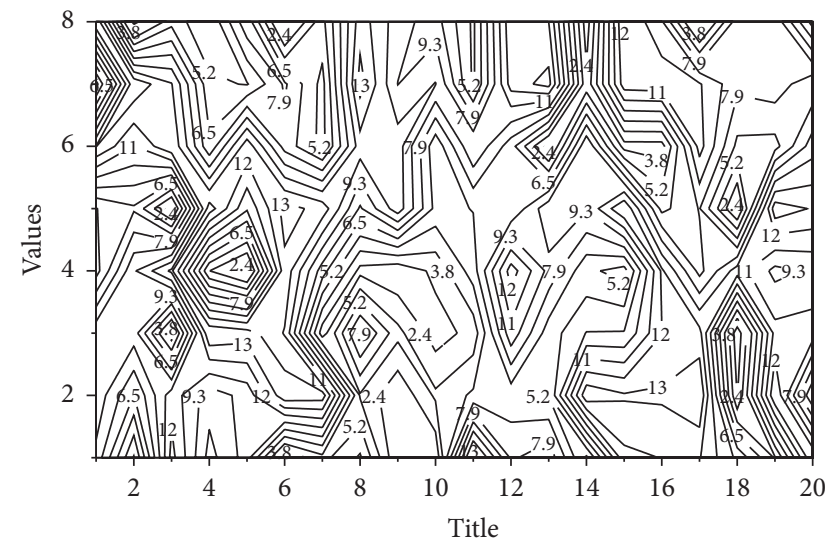

Figure 7: Pre-test statistics.

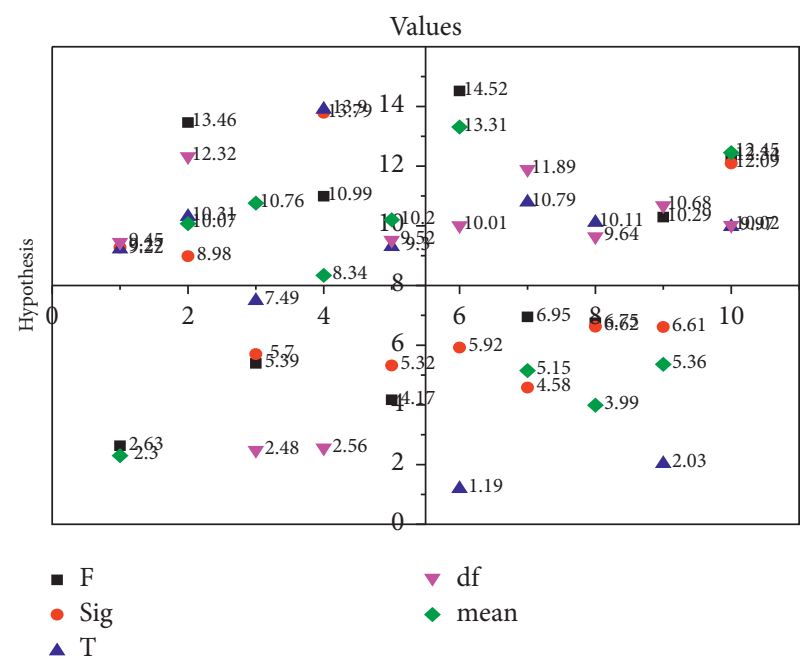

Figure 8: T-test results.

\section{Conclusion}

The use of micro-video scaffolding in the junior high school English listening classroom can significantly improve students' learning performance. Analysis of the pre- and posttest scores revealed that although both classes improved their English listening test scores in the first semester compared to the pre-experimental period, the further comparison revealed that the experimental class students' English listening scores improved more. In addition, the interview with the students of the experimental class revealed that the students of the class were generally more active in completing the tasks assigned by the teacher and were also able to participate in learning activities more actively and positively. What is more important is that both classes used the same teaching materials, but the only difference was the teaching method. This shows that the use of micro-video scaffolding teaching in junior high school English listening classrooms can stimulate students' motivation to learn English listening, improve learning efficiency, and eventually improve listening performance. The only difference between the two classes is the teaching method.
This shows that the use of micro-video scaffolding in junior high school English listening classroom teaching can motivate students to learn English listening, improve learning efficiency, and eventually improve listening performance. The results of the students' reading motivation questionnaire after using the hybrid teaching model of micro-videoscaffolded classroom show that students' English reading motivation in the three dimensions of learning attitude, learning reasons, and learning behavior has been improved and maintained at a high level, mainly because students are more interested in learning English reading, learning English reading more out of deep internal learning motivation, completing the reading task on time, and taking the initiative to read outside the classroom.

\section{Data Availability}

The data used to support the findings of this study are included within the article.

\section{Conflicts of Interest}

No conflicts of interest exist concerning this study.

\section{Acknowledgments}

This paper was not funded by any organization.

\section{References}

[1] Z. Xu and Y. Shi, "Application of constructivist theory in flipped classroom-take college English teaching as a case study," Theory and Practice in Language Studies, vol. 8, no. 7, pp. 880-887, 2018.

[2] X. Ma, Y. Su, J. Liu, and S. Li, "Design and test of flipped classroom learning support model in mobile learning environment," Creative Education, vol. 10, no. 2, pp. 246-261, 2019.

[3] Y. Wang, "A study on college English high-efficiency class based on blended teaching mode of flipped classroom," Theory and Practice in Language Studies, vol. 10, no. 9, pp. 1066-1071, 2020.

[4] J. Qin and Z. Jia, "Learner-generated behaviours in a flipped learning: a focus on computer culture foundation course," International Journal of Continuing Engineering Education and Life Long Learning, vol. 30, no. 1, pp. 1-14, 2020.

[5] A. D. Fang, G. L. Chen, Z. R. Cai, L. Cui, and L. Harn, "Research on blending learning flipped class model in colleges and universities based on computational thinking- "Database principles" for example," Eurasia Journal of Mathematics, Science and Technology Education, vol. 13, no. 8, pp. 57475755, 2017.

[6] J. Liu, "The implementation of flipped classroom teaching mode in basic English teaching for Tibetan college students," Theory and Practice in Language Studies, vol. 9, no. 8, pp. 1009-1014, 2019.

[7] F. Jin, "Exploring the blended teaching mode under the guidance of OBE theory-taking the course of English newspaper reading $I$ in Zhejiang Yuexiu university as an example," Open Journal of Modern Linguistics, vol. 11, no. 4, pp. 511-519, 2021. 
[8] D. Zhao and X. Liang, "The research on the professional development of information technology teachers based on the implementation of flipped classroom teaching model," International Journal of Social and Humanistic Computing, vol. 3, no. 2, pp. 176-190, 2019.

[9] M. Chen, B. Li, Y. Hu, and Z. Ren, "A teaching ecosystem of engineering graphics based on the deeper learning cycle model and asynchronous SPOC," Journal for Geometry and Graphics, vol. 23, no. 2, pp. 259-267, 2019.

[10] Z. Fan and F. Shu-Xiong, "The process of personalized learning based on flipped classroom," Sino-US English Teaching, vol. 14, no. 4, pp. 233-238, 2017.

[11] N. Gao, "Construction and implementation of teaching mode for digital Mapping based on interactive micro-course technology," International Journal of Emerging Technologies in Learning (iJET), vol. 13, no. 2, pp. 21-32, 2018.

[12] M. Huang and C. Lijian, "The path of building curriculum resources of adult colleges and universities based on MOOC in the intelligent era," Canadian Social Science, vol. 16, no. 6, pp. 32-38, 2020.

[13] W. Zhang and L. E. Vesnina, "Online teaching Russian practical grammar at a Chinese university during the epidemic," Russian Language Studies, vol. 18, no. 4, pp. 383-408, 2020.

[14] Z. Wei, "Discussion on the effective application of information technology in informatization EFL teaching," Theory and Practice in Language Studies, vol. 8, no. 10, pp. 1294-1300, 2018.

[15] J. Xiao, M. Cao, X. Li, and P. Hansen, "Assessing the effectiveness of the augmented reality courseware for starry sky exploration," International Journal of Distance Education Technologies, vol. 18, no. 1, pp. 19-35, 2020.

[16] J. Cai, S. Zhu, Y. M. Tian, and H. H. Yang, "Learning from practice: improving blended learning strategies in an educational technology course," International Journal of Innovation and Learning, vol. 21, no. 4, pp. 467-480, 2017.

[17] S. Shi and Z. A. B. Zainuddin, "A review of the research on blended learning in physical education in China and international," Journal of Critical Reviews, vol. 7, no. 11, pp. 672-686, 2020.

[18] P. J. García Nieto, E. García-Gonzalo, J. P. Paredes-Sánchez, A. Bernardo Sánchez, and M. Menéndez Fernández, "Predictive modelling of the higher heating value in biomass torrefaction for the energy treatment process using machinelearning techniques," Neural Computing \& Applications, vol. 31, no. 12, pp. 8823-8836, 2019.

[19] L. M. Maiyar, S. Cho, M. K. Tiwari, K.-D. Thoben, and D. Kiritsis, "Optimising online review inspired product attribute classification using the self-learning particle swarmbased Bayesian learning approach," International Journal of Production Research, vol. 57, no. 10, pp. 3099-3120, 2019.

[20] E. M. Golafshani and G. Pazouki, "Predicting the compressive strength of self-compacting concrete containing fly ash using a hybrid artificial intelligence method," Computers and Concrete, vol. 22, no. 4, pp. 419-437, 2018.

[21] F. Daneshfar and S. J. Kabudian, "Speech emotion recognition using discriminative dimension reduction by employing a modified quantum-behaved particle swarm optimization algorithm," Multimedia Tools and Applications, vol. 79, no. 1, pp. 1261-1289, 2020. 